\title{
Activation of reward circuitry in human opiate addicts
}

\author{
L. A. Sell, J. Morris, ${ }^{1}$ J. Bearn, R. S. J. Frackowiak, ${ }^{1}$ K. J. Friston ${ }^{1}$ and R. J. Dolan ${ }^{1}$ \\ National Addiction Centre, 4 Windsor Walk, London SE5 8AF, UK \\ ${ }^{1}$ Wellcome Department of Cognitive Neurology, Institute of Neurology, London WC1N 3 BG, UK
}

Keywords: addiction, cue, heroin, neuroimaging

\begin{abstract}
The neurobiological mechanisms of opiate addictive behaviour in humans are unknown. A proposed model of addiction implicates ascending brainstem neuromodulatory systems, particularly dopamine. Using functional neuroimaging, we assessed the neural response to heroin and heroin-related cues in established opiate addicts. We show that the effect of both heroin and heroin-related visual cues are maximally expressed in the sites of origin of ascending midbrain neuromodulatory systems. These context-specific midbrain activations predict responses to salient visual cues in cortical and subcortical regions implicated in reward-related behaviour. These findings implicate common neurobiological processes underlying drug and drug-cue-related effects.
\end{abstract}

\section{Introduction}

In humans the biological mechanisms through which drugs of addiction and drug-related cues exert their effects are largely unknown. Neurobiological processess which are well described in animals have yet to be demonstrated in humans. We address this question, using functional neuroimaging, by studying opiate addicts during exposure to heroin and heroin-related visual cues. Our experimental design enabled us not only to examine for the effect of the drug but also drug cues and their interaction. Using a novel data-led approach we were also able to assess how a region of activation, common to the effects of both drug and drug cues, selectively influenced the processing of salient drug cues.

An important neurobiological model of opiate addiction dissociates neural systems mediating positive from those mediating negative reinforcement (Wise \& Bozarth, 1987). The locus coeruleus and periaqueductal grey matter in the brainstem are implicated in the opiate withdrawal syndrome, i.e. negative reinforcement (Legradi et al., 1996). Neural systems implicated in reward or positive reinforcement include the brainstem ventral tegmental area (VTA) and its target regions, which include ventral striatum as well as limbic, cingulate and prefrontal cortex (Kim et al., 1986; Wise, 1987; Schulteis \& Koob, 1994; DiChiara, 1995; Altman et al., 1996; Masterman \& Cummings, 1997). The 'psychomotor stimulant' and 'incentive motivation' theories of addiction describe a central role of ascending dopamine pathways in this mesolimbocortical system in driving drug-seeking behaviour.

An important pharmacological effect of heroin is disinhibition of VTA $\gamma$-aminobutyric acid (GABA) interneurons. This results in increased firing of VTA dopaminergic neurons (DiChiara \& North, 1992; Koob, 1992a; Wise, 1996; Schultz et al., 1997) and thus activation of the mesolimbic dopamine system. Behavioural models of reward, including intracranial self stimulation and conditioned place preference, also implicate the mesolimbic dopamine system in

Correspondence: Dr L. A. Sell, Drugs North West, Mental Health Services of Salford, Bury New Road, Manchester M25 3BL, UK.

E-mail: Louohn@aol.com

Received 19 March 1998, revised 16 July 1998, accepted 30 October 1998 addiction (Schultz, 1992; Shippenberg et al., 1992; Wise, 1992). The reinforcing effects of opiates can be ascribed to direct pharmacological actions (unconditioned stimulus, UCS) or to the effects of stimuli that have acquired salience through temporal association with drug exposure (conditioned stimulus, CS) (Robinson \& Berridge, 1993). Cues associated with drug use may thus elicit conditioned craving, as well as conditioned drug and conditioned-withdrawal-like effects (Childress et al., 1986). In animals, exposure to stimuli previously associated with drugs increases the firing of VTA dopaminergic neurons (Schultz, 1992), potentiates release of dopamine in the nucleus accumbens (Fontana et al., 1993) and modulates cerebral activity in target areas of the ascending dopamine system (Trusk \& Stein, 1988). These effects mirror increased dopamine release in the nucleus accumbens in response to cues predicting ingestive and sexual reward (Phillips et al., 1991).

Interactions between neuromodulatory dopaminergic systems ascending from the brain stem, and target limbic regions are critical in addictive behaviour (Robbins et al., 1989; Robbins \& Everitt, 1996). The meso-amygdaloid system has a crucial role in acquisition of addictive behaviour due to its role in the formation of associations between UCS and CS (Cador et al., 1989; Williams et al., 1993; Whitelaw et al., 1996). It could be expected to have a central role in the aquisition of salience by opiate-related cues. In addition to the well established role of dopamine, reward mechanisms have been demonstrated to involve nondopamine-dependent processes (Koob, 1992b).

The present study was designed to explore the relevance of these findings in real life situations by studying heroin addicts during exposure to both drug-salient cues and heroin. We used positron emission tomography (PET) to index neural activity in a $2 \times 2$ factorial design, with the presence or absence of heroin as one factor, and the presence or absence of salient visual cues as a second factor. We predicted that heroin exposure would result in activation of the mesolimbic dopamine system. Secondly, on the basis of single cell neurophysiology data (Schultz et al., 1997) we predicted that salient cue exposure would result in a similar pattern of activation. Our experimental design also allowed us to examine interactions between heroin and salient cues. Thirdly, we predicted that activation of the 
TABLE 1. Study design. Subjects underwent six scans on each day, watching a video during each scan, and on each day receiving a single injection of heroin or placebo after the second scan and ratings. Therefore scans 3-6 are designated as placebo or heroin condition. Visual analogue scales ('Ratings') were measured at baseline and immediately after each scan (see Figs 1 and 2). The results of these are in Figs 1 and 2 . The order of Days A and B was randomly counterbalanced.

\begin{tabular}{|c|c|c|c|c|c|c|c|}
\hline Scans & Base-line & 1 & 2 & 3 & 4 & 5 & 6 \\
\hline 'Ratings' & 'B' & 'n1' & 'd1' & 'n2' & 'd2' & 'n3' & 'd3' \\
\hline \multicolumn{8}{|l|}{ Day A } \\
\hline Video condition & & Neutral & Salient & Neutral & Salient & Neutral & Salient \\
\hline Drug condition & & Pre-injection & Pre-injection & Placebo & Placebo & Placebo & Placebo \\
\hline \multicolumn{8}{|l|}{ Day B } \\
\hline Video condition & & Neutral & Drug & Neutral & Drug & Neutral & Drug \\
\hline Drug condition & & Pre-injection & Pre-injection & Heroin & Heroin & Heroin & Heroin \\
\hline
\end{tabular}

ascending neuromodulatory systems would enhance neural responses to salient visual stimuli (i.e. to a drug video) in the brain systems implicated in reward. We tested this third prediction by examining for an interaction between brainstem activity and the presence or absence of saliency in the visual stimuli (drug versus neutral video). This constitutes a psychophysiological interaction and was tested for with statistical parametric mapping (SPM) and suitable regressors, as described previously (Friston et al., 1997).

\section{Methods}

Subjects were 10 male in-patients at the detoxification unit of the Bethlem Hospital. All were current heroin injectors over 18 years of age, and gave informed consent to the procedure which was approved by the local hospital ethics committee. Serious psychiatric or medical illness, alcohol dependence in the last two years, or a history of cocaine or amphetamine dependence, resulted in exclusion. Mean age was 31.6 years (range 23-39 years). All were white Caucasian. Mean length of heroin use was 12.4 years (range 2-19 years), of dependence 8.7 years (range 2-16 years) and of injecting drugs 9.5 years (range 1-18 years). They had a mean of 2.8 previous in-patient detoxification treatments (range 0-5). Eight received a prescription for methadone prior to admission (mean daily dose $28.75 \mathrm{mg}$; range $15-50$ years). All used heroin and two used illicit methadone. The total daily methadone equivalent dose, taking account of prescribed and illicit methadone and heroin, and assuming $1 \mathrm{~g}$ of street heroin is equivalent to $60 \mathrm{mg}$ of methadone, was $54 \mathrm{mg}$ (range $27-110 \mathrm{mg}$ ). Subjects injected a mean of 2.89 times daily (range 1-6 times). Seven currently used diazepam, one on a daily basis. The mean weekly dose, including prescribed and illicit sources, of those who used diazepam was $213 \mathrm{mg}$ (range 37.5-750 mg). All had drunk alcohol, and three did so currently. All had used cannabis, of whom, five still did. All had used amphetamine but none did so currently. Eight subjects had used cocaine, of whom, seven still did on rare occasions. Seven had used LSD and seven ecstasy; neither drug was currently used by any subject. No subject used: barbiturates, although four had in the past; solvents, although three had in the past; or other opiates, although three had in the past.

Subjects underwent methadone dose titration, and stabilization using single blind methadone administration occurred on days 1 and 2 following admission to hospital. Four subjects who were codependent on benzodiazepines were also stabilized on diazepam.

Subjects had a magnetic resonance scan (MRI) on day 3. PET scans were performed on days 3 and 5, according to the design shown in Table 1, counterbalancing for order of placebo and heroin. At 8.30 am on scanning days, subjects were given $50 \%$ of the total methadone dose they had consumed during the previous $24 \mathrm{~h}$. Diazepam was maintained at a constant dose for the duration of the study. During
PET data aquisition, subjects viewed, alternatively, a colour video of a volunteer assembling a neutral household object and a salient colour video showing a different volunteer preparing and administering heroin by injection. Each video lasted $5 \mathrm{~min}$ and PET data aquisition started during the final $40 \mathrm{~s}$ of the video.

'Ratings' data were gathered during the $3 \mathrm{~min}$ which elapsed between each video, according to the cyclotron activity cycle. The ratings were subjective measures using verbal analogue scales (VAS) (1-10) of; craving for heroin (VAS 'craving'), desire for a hit (VAS 'hitdesire'), desire to leave and score (VAS 'hitdrive'), feeling high (VAS 'high'), withdrawal symptoms (VAS 'with'), feeling happy (VAS 'happy'), feeling tense, (VAS 'tense') (Sell et al., 1995). These measures were taken at baseline and after each scan, in order to calculate a change from baseline for each scan. A composite measurement 'urge to use' was calculated by summing the change from baseline responses to the VASs 'craving', 'hitdesire' and 'hitdrive', giving a possible maximum 'urge' rating of 30 .

Each subject was given placebo or heroin after the second scan and video. Subject and scanning staff were kept strictly blind to the condition. The only person who knew was the administering doctor who prepared and gave a single intravenous bolus, following the same procedure and using the same words on each occasion. Thus the subject knew they were receiving an injection but were reminded that it could be placebo or heroin each time. The heroin dose for each patient was predetermined according to a sliding scale from $10 \mathrm{mg}$ to $30 \mathrm{mg}$ depending on their methadone dose, but in the event all received $20 \mathrm{mg}$.

PET data acquisition and analysis was performed using methods described previously (Talairach \& Tournoux, 1988; Friston et al., 1991; Friston et al., 1995a,b). Scans of the distribution of $\mathrm{H}_{2}{ }^{15} \mathrm{O}$ were obtained using a Siemens/CPS ECAT EXACT HR + PET Scanner operated in high sensitivity 3D mode. Subjects received a total of $350 \mathrm{Mbq}$ of $\mathrm{H}_{2}{ }^{15} \mathrm{O}$ intravenously over $20 \mathrm{~s}$ through a forearm cannula for each of the 12 scans. Integrated activity was measured over a 90-s time window. The PET images comprised i, j, and $\mathrm{k}$ voxels $(2 \times 2 \times 3 \mathrm{~mm})$ with a $6.4-\mathrm{mm}$ transaxial and $5.7 \mathrm{~mm}$ axial resolution (full width at half maximum). The data were analysed with SPM (SPM96, Wellcome Department of Cognitive Neurology, London) implemented in Matlab (Mathworks, Sherborn, MA, USA). Structural $\mathrm{T}_{4}$-weighted MRIs from each subject were coregistered to the PET data following realignment of the PET time series (Friston et al., 1995b). All scans were then transformed into a standard stereotactic space (Talairach \& Tournoux, 1988). The scans were smoothed using a Gaussian filter (12 mm full width at half maximum). A blocked (by subject) ANCOVA model was fitted to the data at each voxel, with condition effects for the drug and video experimental variables, and global CBF as a confounding covariate (Friston et al., 1995a). Predetermined contrasts of the condition effects at each voxel 
were assessed using the usual $t$-statistic, giving an SPM map for each contrast (Friston et al., 1991). The general methods employed by SPM are described in detail by Friston et al. (Friston et al., 1995b).

In the regression analysis, the regional $\mathrm{CBF}(\mathrm{rCBF})$ values at the maximal focus of activation in the midbrain in the drug main-effect analysis, were grouped into pairs of covariates corresponding to the experimental conditions, i.e. drug video and neutral video. These sets of values were then used as covariates of interest in SPM96 to produce a separate analysis of psychophysiological interactions (Friston et al., 1997). Differences between the regression slopes obtained for the

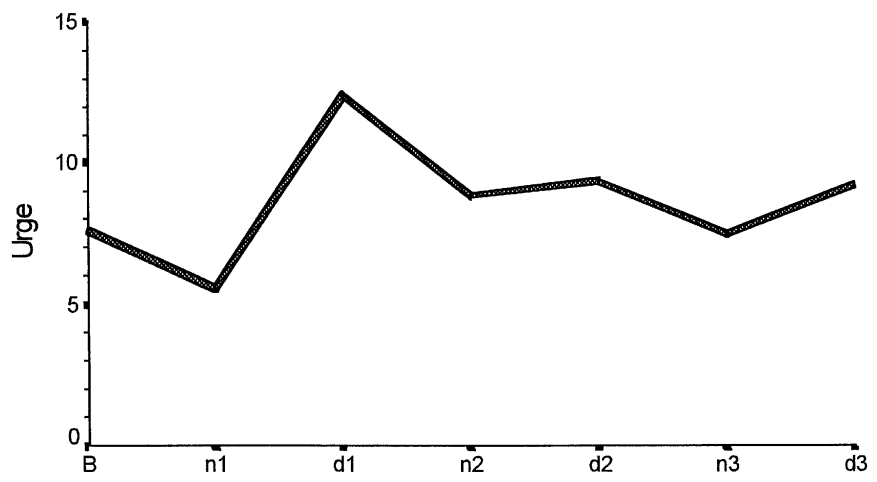

Ratings at baseline and during scans 1 to 6

FIG. 1. Urge to use heroin. The subjective urge to use heroin obtained by summing responses to three visual analogue scales rated at baseline (B) and after each scan ( $\mathrm{n} 1$ to $\mathrm{d} 3, \mathrm{n}=$ neutral cue; $\mathrm{d}=$ drug-salient cue).

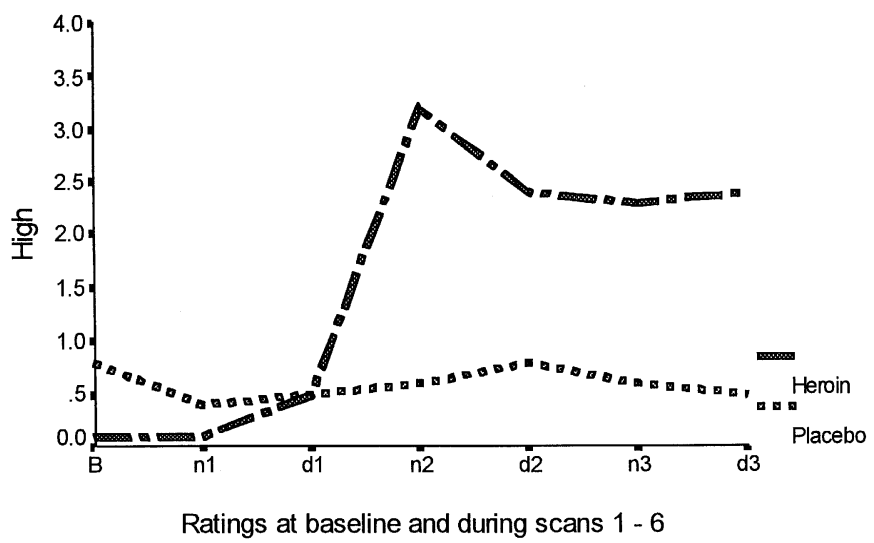

FIG. 2. Feeling high. The subjective report of feeling high obtained by rating responses on a visual analogue scale at baseline (B) and after each scan (n1 to $\mathrm{d} 3, \mathrm{n}=$ neutral cue; $\mathrm{d}=$ drug-salient cue). Responses in placebo and heroin sessions are displayed separately, reflecting the significant effect of the drug (see text). pairs of covariates at each voxel in the brain were tested directly in SPM96 to produce a $\operatorname{SPM}(t)$ showing voxels in which the contribution of midbrain activity changed significantly as a function of experimental condition.

\section{Results}

Figures 1 and 2 show the self report of urge to use heroin and feeling high across the scanning sessions. The subject self report data, rated at baseline and after each scan on a visual analogue scale, demonstrated the effectiveness of the experimental design. Repeated measures analysis of variance indicated an effect of heroin on the report of feeling high $\left(F_{2}=12.65, P<0.01\right)$. Injection of heroin was associated with reports by subjects that they felt high. There was no significant effect on the subjects' self report of symptoms of withdrawal. The salient visual cue (video) had a significant effect on the composite rating of reported urge to use heroin $\left(F_{1}=14.11\right.$, $P<0.01)$; there was also an effect of session order on urge to use $\left(F_{1}=6.34, P<0.05\right)$ and an interaction of video by time $\left(F_{1}=\right.$ 7.84, $P<0.05)$. Exposure to this set of conditioned cues therefore was associated with a reported urge to use heroin. The effect of time may reflect habituation to the repeated videos. This observation merits consideration quite apart from the scan data reported here and will be discussed in detail elsewhere.

The contrasts of condition-specific effects employed in analysing the scan data tested for the main effects and interactions, with results reported at an uncorrected level in brain regions where activations were predicted. Unpredicted activations are reported only if they survived correction for the effect of multiple comparisons in the entire brain volume (see Fig. 3 and Table 2). Heroin administration resulted in significant activation $(Z=4.37, P<0.001$, uncorrected) in the midbrain within an area centred on the periaqueductal grey but

TABLE 2. Stereotactic co-ordinates and statistical significance of brain activity in the contrasts comparing the heroin and placebo conditions, comparing the salient video and nonsalient video conditions, and showing the interaction between heroin and salient video conditions.

\begin{tabular}{lrrrrl}
\hline Brain area & $x$ & $y$ & $\mathrm{z}$ & $Z$-score & $P$-value \\
\hline $\begin{array}{l}\text { Heroin condition } \\
\quad \text { Midbrain }\end{array}$ & 0 & -26 & -12 & 4.37 & $<0.001^{*}$ \\
$\begin{array}{l}\text { Salient video condition } \\
\quad \text { Midbrain }\end{array}$ & 0 & -22 & -6 & 4.47 & $<0.001^{*}$ \\
$\quad$ Insula & -38 & 10 & -8 & 4.56 & $<0.05^{*}$ \\
$\quad$ Interaction heroin and salient video condition & & & \\
$\quad$ Parietal & 24 & -42 & 46 & 3.29 & $>0.05 \dagger$ \\
$\quad$ Parietal & -42 & -52 & 32 & 3.49 & $>0.05 \dagger$ \\
\hline
\end{tabular}

$* P$ uncorrected; $† P$ corrected for whole brain comparison.

FIG. 3. SPMs of midbrain activations in the contrasts of (A) heroin versus placebo and (B) drug video versus neutral video conditions. The SPMs are displayed on coronal slices of a canonical MRI image at (A) $y=-26 \mathrm{~mm}$ and (B) $y=-24 \mathrm{~mm}$, with a threshold of $P<0.01$ for both contrasts. Mean adjusted rCBF $(\mathrm{mL} / \mathrm{dL} / \mathrm{min})$ in the maximally activated voxels $(x=0, y=-26, z=-10$ in (A), and $x=0, y=-24, z=-6$ in (B) are shown graphically for (C) predrug, postheroin and postplacebo conditions, and (D) neutral and drug video conditions. Bars represent 2 SDs.

FIG. 4. SPMs and regression plots showing psychophysiological interactions between right anterior cingulate cortex and midbrain. rCBF values from the midbrain voxel $x=0, y=-26, z=-10$ were used to produce condition-specific predictor variables (i.e.separate covariates for drug video and neutral video conditions) and the regression with the rCBF in all other brain voxels, for each condition, was calculated and contrasted in SPM96. The resulting SPMs show that the midbrain augments or modulates anterior cingulate, left extended amygdala/medial basal forebrain and left dorsolateral prefrontal cortex responses during the drug video condition (relative to the neutral video). A threshold of $P<0.01$ was used for the SPM, which is displayed on a sagittal $(x=6 \mathrm{~mm})$ slice of a canonical MRI image. The condition-specific changes in the contribution of the midbrain to anterior cingulate activity are illustrated by bivariate regression plots of $\mathrm{rCBF}(\mathrm{mL} / \mathrm{dL} / \mathrm{min})$ from midbrain $(x=0, y=-26, z=-10)$ and right anterior cingulate $(x=6, y=34, z=6)$ voxels. Broken lines represent $95 \%$ confidence limits for the regression slope. Only in the drug video conditions is there a significantly positive regression between midbrain and anterior cingulate activity. 


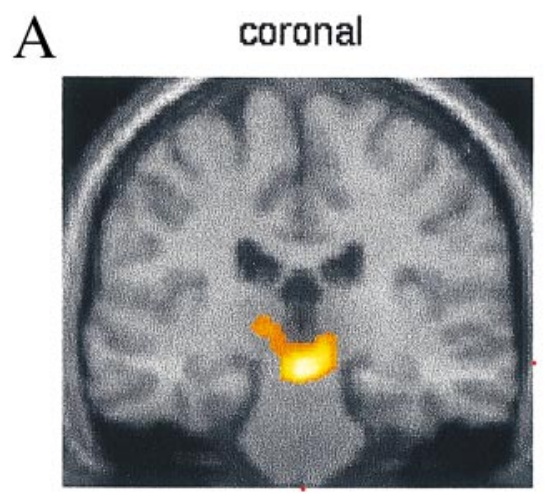

C

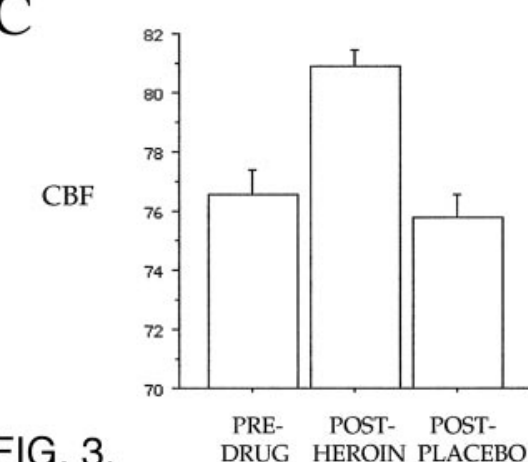

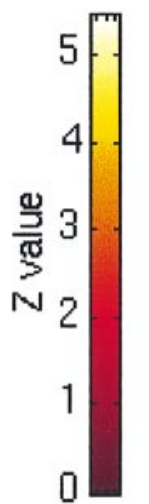

B

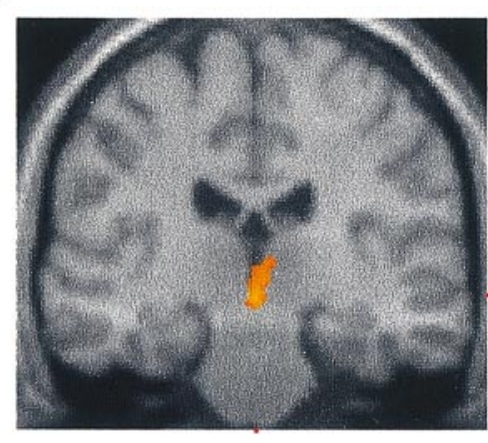

D

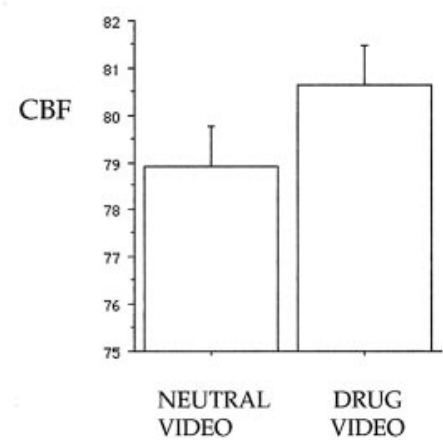

B
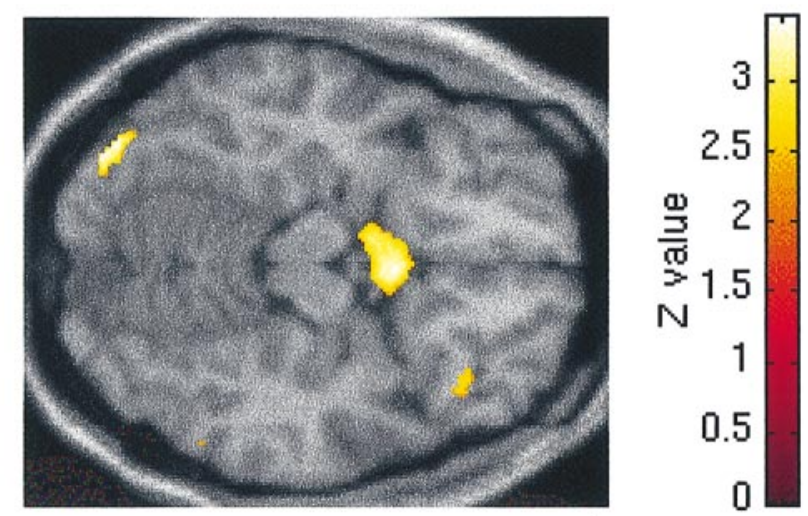

D

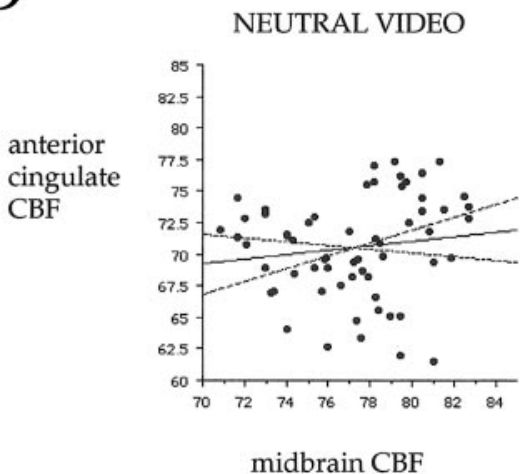

midbrain $\mathrm{CBF}$
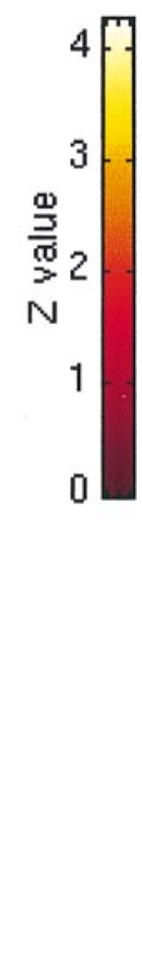

A

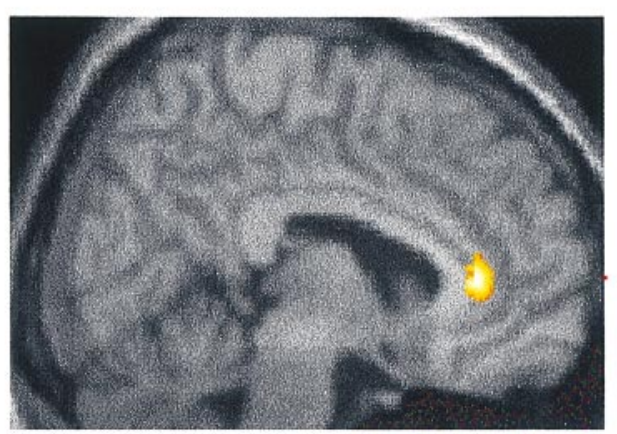

C

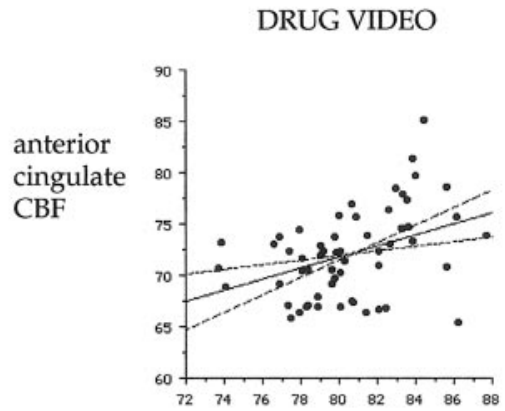

FIG. 4.

midbrain $\mathrm{CBF}$ 
TABLE 3. Stereotactic coordinates and statistical significance of brain activity in the contrasts comparing the salient video and neutral video conditions, with the values at the midbrain voxel, $x=0, y=-26, z=-10$, as covariate of interest.

\begin{tabular}{lrrrrr}
\hline Brain area & $x$ & $y$ & $\mathrm{z}$ & $Z$-score & $P$-value \\
\hline Main effect salient video & & & & & \\
$\quad$ Pre-frontal & -48 & 8 & 24 & 3.95 & $<0.001$ \\
$\quad$ Basal forebrain & -8 & 0 & -16 & 3.22 & $<0.001$ \\
Anterior cingulate & 6 & 36 & 8 & 3.46 & $<0.001$ \\
\hline
\end{tabular}

The $P$-value reported is uncorrected.

extending to include regions such as VTA and dorsal raphe. The salient drug video compared with the neutral video resulted in activation in similar and overlapping brain midbrain regions $(Z=$ $3.82, P<0.001$, uncorrected) to those of heroin itself. An additional focus of activation was found in the left insula $(Z=4.56, P<0.05$, corrected) as well as in both cerebellar hemispheres (right: $Z=5.42$, $P<0.05$, and left: $Z=4.55, P<0.05$, both corrected). There was no interaction between heroin and the salient visual stimuli that survived correction although we report an interaction in both medial parietal cortices $(P<0.001$, uncorrected) for descriptive purposes. There was no effect on blood flow in the temporal lobe by either heroin or salient video.

Results of the psychophysiological interaction are shown in Fig. 4 and Table 3. The interaction defined in our factorial experiment allows us to investigate modulation. Modulation refers to a change in response to an afferent input without alteration in basal neuronal firing levels. Regions of significant activation here indicate areas whose actvity in response to the salient video is modulated by activity in midbrain ascending pathways in response to heroin adminstration. A significant modulatory effect was found in the anterior cingulate, the left dorsolateral prefrontal cortex and the left extended amygdala/ basal forebrain region. These areas show a greater response to the salient over the neutral video in proportion to activation in the midbrain during the heroin as opposed to the placebo condition.

\section{Discussion}

The effects of heroin and the drug video were both centred on the midbrain in an area that has widespread and reciprocal connections with regions that support reward-related behaviour and emotional learning (Bragin et al., 1984; Wyss \& Sripanidkulchai, 1984; Li et al., 1989; Meller \& Dennis, 1991; Rizvi et al., 1991; Fiorino et al., 1993). We suggest that the focus of activation for the two main effects appears to be in the periaqueductal grey, but it spreads to include the VTA. The smoothing procedure in the data analysis means that activations in the periaqueductal grey and VTA could not be confidently resolved. Therefore we need to consider both of these midbrain regions. The VTA contains the dopaminergic cell bodies which project via the mesolimbic dopamine system, as described in the introduction. The periaqueductal grey has projections to the VTA, central and basolateral nuclei of amygdala (Meller \& Dennis, 1991). It receives afferents from cingulate, insular and medial prefrontal cortices (Bragin et al., 1984; Wyss \& Sripandkulchai, 1984). The fact that pharmacological challenge and salient visual stimulation activate similar brain systems is congruent with predictions from theoretical accounts of value-dependent reinforcement learning (Friston et al., 1994). In this instance, heroin can be thought to have intrinsic, or innate, value by virtue of direct access to the ascending midbrain systems. Conversely, the drug video embodies stimuli that have acquired value. In this model of reinforcement learning, the effect of activation of ascending neuromodulatory systems would be, directly or indirectly, to consolidate changes in synaptic plasticity that underly the learning of addictive behaviour.

The ascending midbrain systems also exert a more classical neuromodulatory effect. To demonstrate these effects we predicted that activation of ascending neuromodulatory systems would enhance neural responses to salient visual stimuli (i.e. the drug video) in those brain systems implicated in the processing of hedonic or valuable stimuli. We used measured brainstem activation as a direct index of activity in ascending neuromodulatory systems, and tested the prediction by examining for an interaction between brain stem activity and the presence or absence of saliency in the visual stimuli (drug versus neutral video). This constituted a psychophysiological interaction. The critical response characteristic from this analysis is that anterior cingulate, amygdala/basal forebrain and dorsalateral prefrontal cortex activity discriminates positively between salient and nonsalient visual cues when, and only when, activity in the midbrain area is high. Such a result is predicted if modulatory afferents from the midbrain augment neural responses to valuable stimuli in processing regions that are its projection targets. A psychophysiological interaction allows inferences beyond those that can be drawn from psychopharmacological interactions (Friston et al., 1992) by implicating the behaviour for which the pharmacological output, in this instance processing of saliency, is relevant.

Our data confirm that both heroin and drug-related visual cues are associated with a neural response in similar midbrain regions. An effect of heroin in this region can be explained as a direct unconditioned effect of the drug in an area known to be rich in opiate receptors (Koob, 1992; Legradi et al., 1996). Strikingly, the visual drug cue elicited a neural response similar to that of heroin. We suggest that this effect probably reflects a direct influence, on this region, by regions involved in the processing of visual drug cues. Similar effects have been reported when a previously neutral stimulus acquires behavioural salience (Morris et al., 1997).

Pairing a CS with an UCS results in the CS eliciting firing of DA neurons (Williams et al., 1993; Schultz et al., 1997). The finding of a response to heroin in the periacqueductal grey (PAG) and surrounding regions that mirrored the response to a salient cue (i.e. CS) strongly suggests that these effects in the PAG are related to conditioned processes intimately involved in reward dependence. These effects may be mediated via known reciprocal connectivity between the VTA and PAG (Meller \& Dennis, 1991). The other region of significant activation in response to a salient visual cue was that of the insula. The insula has been proposed as a limbic integration cortex and in the present context it may integrate cues with their emotional relevance. We suggest that activation in this region may also reflect somatic components of the anticipatory anxiety experienced by subjects when exposed to a salient visual cue (Mesulum, 1985).

A complex neural system involving the limbic-motor interface of the ventral-striatal-pallidal system has been suggested by animal models of addiction and reward dependence (Altman et al., 1996; Robbins \& Everitt, 1996). A parallel system subserving opiate withdrawal has long been recognized (Wise, 1987; Legradi et al., 1996). Models in which the activity of these two systems are seen as distinct may be too simplistic (Maldonado et al., 1997; Cazala, 1984). In our study both drug and salient cues resulted in very similar direct effects on dopaminergic target sites (Bjorkland \& Lindval, 1984; Kim et al., 1986; DiChiara, 1995) that are also implicated in motivational and reward-related mechanisms. However, our data have wider implications in that they suggest that opiate reward dependence could involve not only ascending brainstem pathways but also important inputs from the PAG. Dependence on opiate-related rewards 
(opiate dependence) may thus involve critical interactions between systems mediating opiate withdrawal and those mediating positive reinforcement.

A key feature of our experimental design is that it emulates, as closely as possible, real life scenarios of dependent addicts who encounter all the modelled combinations of presence or absence of drug and drug-related cues. It may be that our design allowed insufficient time for those subjects who had been using mostly heroin (and little or no methadone) in a particularly chaotic manner prior to admission to stabilize. However, the ward protocol is designed to titrate and stabilize patients rapidly prior to detoxification. Having had $50 \%$ of their previous day's dose $6 \mathrm{~h}$ before the commencement of the scanning procedure, patients were neither intoxicated nor in withdrawal. Lack of stability would be expected to introduce excessive variance into the data, and reduce the likelihood of statistically significant results. It is possible that our findings were also influenced by other clinical variables such as benzodiazepine codependence. One way of controlling for these confounding factors is to study abstinent addicts. There are ethical concerns about exposing abstinent addicts to heroin, because of the risk of relapse. Restricting our study to current addicts allows the synchronized investigation of both cue exposure and heroin adminstration. Our model specifically subtracts placebo from heroin and neutral video from salient video effects, using each subject as their own control, and it allows us to identify statistically significant effects of heroin injection and salient video presentation despite the many other variables which exist in both our design and the neuropsychological systems of active addicts. The approach we describe here provides important information about the neuropsychological processes in addicts actively using drugs, the majority of whom, when encountered in clinical and real life situations, are polydrug addicts. Consequently, our results have a high ecological validity, and they provide, for the first time, evidence in humans for a common role of brainstem neuromodulatory systems and their target projection sites in mediating the effects of both heroin itself and salient drug-related cues.

\section{Acknowledgements}

We wish to thank all the patients who participated in this study and Lorraine Wilson and the nursing staff of Wickham Park House, Bethlem Royal Hospital for invaluable help in recruitment and patient care. This research was partly funded by the Wellcome Trust. R.S.J.F., K.J.F., J.M. and R.J.D. are supported by the Wellcome Trust.

\section{Abbreviations}

CS, conditioned stimulus; GABA, $\gamma$-aminobutyric acid; MRI, magnetic resonance imaging; PAG, periacqueductal grey; PET, positron emission tomography; rCBF, regional CBF; UCS, unconditioned stimulus; VAS, verbal analogue scales; VTA, ventral tegmental area.

\section{References}

Altman, J., Everitt, B.J., Glautier, S., Markou, A., Nutt, D., Oretti, R., Phillips, G.D. \& Robbins, T.W. (1996) The biological, social and clinical bases of drug addiction: commentary and debate. Psychopharmacology (Berl.), 125, $285-345$.

Bjorkland, A. \& Lindval, O. (1984) Dopamine containing systems in the CNS In Bjorkland, A.Hokfelt, T. (eds), Handbook of Chemical Neuroanatomy. Vol. 2 (Classical Transmitters in the CNS, 1), pp. 55-122.

Bragin, E.O., Yeliseeva, Z.V., Vasilenko, G.F., Meizerov, E.E., Chuvin, B.T \& Durinyan, R.A. (1984) Cortical projections to the periaqueductal grey in the cat: a retrograde horseradish peroxidase study. Neurosci. Lett., 51, $271-275$.
Cador, M., Robbins, T.W. \& Everitt, B.J. (1989) Involvement of the amygdala in stimulus-reward associations: interaction with the ventral striatum. Neuroscience, 30, 77-86.

Cazala, P. (1984) Electrical self-stimulation of the mesencephalic central gray area: facilitation by lateral hypothalamic stimulation. Physiol. Behav., 32, 771-777.

Childress, A.R., McLellan, A.T. \& O’Brien, C.P. (1986) Conditioned responses in a methadone population. J. Subst. Abuse Treat., 3, 173-179.

DiChiara, G. (1995) The role of dopamine in drug abuse viewed from the perspective of its role in motivation. Drug Alcohol Depend., 38, 95-137.

DiChiara, G. \& North, R.A. (1992) Neurobiology of opiate abuse. Trends Pharmacol. Sci., 13, 185-193.

Fiorino, D.F., Coury, A., Fibiger, H.C. \& Phillips, A.G. (1993) Electrical stimulation of reward sites in the ventral tegmental area increases dopamine transmission in the nucleus accumbens of the rat. Behav. Brain Res., 55, $131-141$.

Fontana, D.J., Post, R.M. \& Pert, A. (1993) Conditioned increases in mesolimbic dopamine overflow by stimuli associated with cocaine. Brain Res., 629, 31-39.

Friston, K.J., Frith, C.D., Liddle, P.F. \& Frackowiak, R.S.J. (1991) Comparing functional (PET) images: The assessment of significant change. J. .Cereb. Blood Flow Metab., 11, 690-699.

Friston, K.J., Grasby, P., Bench, C., Frith, C., Cowen, P.J., Liddle, P., Frackowiak, R.S.J. \& Dolan, R.J. (1992) Measuring the neuromodulatory effects of drugs in man with positron emission tomography. Neurosci. Lett., 141, 106-10.

Friston, K.J., Tononi, G., Reeke, G.N., .Sporns, O. \& Edelman, G.M. (1994) Value-dependent selection in the brain: simulation in a synthetic neural model. Neuroscience, 77, 229-243.

Friston, K.J., Holmes, A.P., Worsley, K., Poline, J.-B., Frith, C.D. \& Frackowiak, R.S.J. (1995a) Statistical parametric maps in functional imaging: a general linear approach. Human Brain Mapping, 2, 189-210.

Friston, K.J., Ashburner, J., Frith, C.D., Poline, J.-B., Heather, J.D. \& Frackowiak, R.S.J. (1995b) Spatial registration and normalization of images. Hum. Brain Mapp., 2, 165-189.

Friston, K.J., Beuchel, C., Fink, G., Morris, J., Rolls, E. \& Dolan, R. (1997) Psychophysiological and modulatory interactions in neuroimaging. Neuroimage, 6, 218-228.

Kim, H.S., Iyengar, S. \& Wood, P. (1986) Opiate actions on mesocortical dopamine metabolism in the rat. Life Sci., 39, 2033-2036.

Koob, G.F. (1992a) Neural mechanisms of drug reinforcement. Ann. NY Acad. Sci., 654, 171-191.

Koob, G.F. (1992b) The dark side of drug dependence. Trends Pharmacol. Sci., 13, 177-184.

Legradi, G.A., Rand, W.M., Hitz, S., Nillni, E.A., Jackson, I.M.D. \& Lechan, R.M. (1996) Opiate withdrawal increases ProTRH gene expression in the ventrolateral column of the midbrain periaqueductal gray. Brain Res., 729, $10-19$.

Li, Y.Q., Rao, Z.R. \& Shi, J.W. (1989) Serotonergic projections from the midbrain periaqueductal gray to the nucleus accumbens in the rat. Neurosci. Lett., 98, 276-279.

Maldonado, R., Salardi, A., Valverde, O., Samad, T.A., Roques, B.P. \& Borrelli, E. (1997) Absence of opiate rewarding effects in mice lacking dopamine D2 receptors. Nature, $\mathbf{3 8 8}, 586-589$.

Masterman, D.L. \& Cummings, J.L. (1997) Frontal-subcortical circuits: the anatomic basis of executive, social and motivated behaviours. $J$. Psychopharmacol., 11, 99-106.

Meller, S.T. \& Dennis, B.J. (1991) Efferent projections of the periaqueductal gray in the rabbit. Neuroscience, 40, 191-216.

Mesulum, M. (1985) Principles of Behavioural Neurology. F.A.Davis, Philadelphia.

Morris, J.S., Friston, K.J. \& Dolan, R.J. (1997) Neural responses to salient visual stimuli. Proc. R. Soc. Lond. B. Biol. Sci., 264, 769-75.

Phillips, A.G., Pfaus, J.G. \& Blaha, C.D. (1991) Dopamine and motivated behaviour: Insights provided by in vivo analyses. In Willner, P.ScheelKruger, J. (eds), The Mesolimbic Dopamine System: from Motivation to Action. Wiley, London, pp. 199-224.

Rizvi, T.A., Ennis, M., Behbehani, M.M. \& Shipley, M.T. (1991) Connections between the central nucleus of the amygdala and the midbrain periaqueductal gray: topography and reciprocity. J. Comp. Neurol., 303, 121-131.

Robbins, T.W., Cador, M., Taylor, J.R. \& Everitt, B.J. (1989) Limbic striatal interactions in reward related processes. Neurosci. Biobehav. Rev., 13, $155-162$.

Robbins, T.W. \& Everitt, B.J. (1996) Neurobehavioral mechanisms of reward and motivation. Curr. Opin. Neurobiol., 6, 228-236. 
Robinson, T.E. \& Berridge, K.C. (1993) The neural incentive basis of drug craving: an incentive sensitization theory of addiction. Brain Res. Rev., 18, 247-291.

Schulteis, G. \& Koob, G. (1994) Dark side of drug dependence. Nature, 371, 108-109.

Schultz, W. (1992) Activity of dopamine neurones in the behaving primate. Semin. Neurosci., 4, 129-138.

Schultz, W., Dayan, P. \& Read Montague, P. (1997) A neural substrate of prediction and reward. Science, 275, 1593-1598.

Sell, L.A., Cowen, P.J. \& Robson, P.J. (1995) Ondansetron and opiate craving: a novel pharmacological approach to addiction. Br. J. Psychiatry, 166 , 511-514.

Shippenberg, T.S., Spanagel, R. \& Herz, A. (1992) Conditioning of opioid reinforcement: neurochemical and neuroanatomical substrates. Ann. NY Acad. S., 654, 347-356.

Talairach, J. \& Tournoux, P. (1988) Co-Planar Stereotaxic Atlas of the Human Brain. Springer Publishing, New York.

Trusk, T.C. \& Stein, E.A. (1988) Effect of heroin conditioned auditory stimuli on cerebral functional activity in rats. Pharmacol. Biochem. Behav., 30, 983-93.

Whitelaw, R.B., Markou, A., Robbins, T.W. \& Everitt, B.J. (1996) Excitotoxic lesions of the basolateral amygdala impair the acquisition of cocaine seeking behaviour under a second order schedule of reinforcement. Psychopharmacology (Berl.), 127, 213-224.

Williams, G.V., Rolls, E.T., Leonard, C.M. \& Stern, C. (1993) Neuronal responses in the ventral striatum of the behaving macaque. Behav. Brain Res., 55, 243-252.

Wise, R.A. (1992) Self-stimulation and drug reward mechanisms. Ann. NY Acad. Sci., 654, 192-198.

Wise, R.A. (1996) Neurobiology of addiction. Curr. Opin. Neurobiol., 6, 243-251.

Wise, R.A. \& Bozarth, M.A. (1987) A psychomotor theory of addiction. Psychol. Rev., 94, 118-132.

Wyss, J.M. \& Sripanidkulchai, K. (1984) The topography of the mesencephalic and pontine projections from the cingulate cortex of the rat. Brain Res., 293, $1-15$. 